Mapping and 5' end determination of kinetoplast maxicircle gene transcripts from Leishmania tarentolae

Agda M.Simpson, Nicolas Neckelmann*, Vidal F.de la Cruz, Michael L.Muhich and Larry Simpson ${ }^{+}$

Department of Biology and Molecular Biology Institute, University of California, Los Angeles, CA 90024, USA

Received 25 March 1985; Revised and Accepted 1 July 1985

\begin{abstract}
Transcripts for six Leishania tarentolae naxicircle structural genes (cytochrome oxidase subunits I, II and III, cytochrone b, human nitochondrial unidentified reading franes 4 and 5 ) and several unidentified open reading franes were napped, and the locations of the $5^{\prime}$ ends deternined by priner runoff analysis. $\Delta 11$ genes studied here are transcribed from the sane strand as the 125 and 95 ribosonal RELs except for the cytochrone oridase subunit I gene. In two cases (ORP3 and ORP4, ORF5 and 0RP6), a single transcript covers two contiguous overlapping reading franes. The 5' ends of the RuAs are located 20-64 nt from the putative translation initiation codons. Prinary transcripts fron a nitochondrial RHA preparatigh were $5^{\prime}$ end-labeled with guanglyltransferase and $\propto-32$-GTP; the aajor labeled species conigrated with the $12 \mathrm{~s}$ and 98 nitochondrial rRMAs, and in addition there were at least four higher nolecular weight labeled species.
\end{abstract}

\title{
IITRODUCTIOF
}

The maxicircle component of the kinetoplast DNA of the hemoflagellate protozoa is homologous to the informational mitochondrial DNA found in other cells (see 1 for review). It consists of a conserved transcribed region containing mitochondrial ribosoma 1 and structural genes, and a non-conserved or 'divergent' region containing tandem repeats of several types (2-5). The complete sequences of the transcribed regions of maxicircle DNA from two species, Leishmania tarentolae and Trypanosoma brucei, are known (6-11 and Neckelmann, de la Cruz and Simpson, unpublished results). The genes for cytochrome oxidase subunits I (COI) and II (COII), cytochrome $B$ ( $C Y b)$, and human mitochondrial unidentified reading frames 4 (HORF4), 5 (HURF5) and 1 (HORF1) have been identified in both species, in addition to several unidentified reading frames. The L. tarentolae maxicircle also contains the gene for 
cytochrome oxidase subunit III (COIII), which is not found in the T. brucei sequence.

The conserved region of the maxicircle DNA is transcribed extensively. Preliminary transcription maps have been constructed for both L. tarentolae and $\underline{T}$. brucei by probing RNA blots with labeled restriction fragments, and tentative candidates for transcripts of several specific genes have been proposed $(9,10,12-16)$.

In this paper we identify specific transcripts of the known structural genes of the $\underline{\text { L }}$ tarentolae maxicircle and localize the $5^{\prime}$ ends of these transcripts by primer runoff experiments.

\section{MATBRIALS ATD MBTHODS}

\section{Cel1 culture and isolation of kinetoplast fraction}

L. tarentolae cells were grown in Difco Brain Heart Infusion medium with hemin at 27 C as described (17). A purified kinetoplast-mitochondrion cell fraction was isolated from early 108 phase cells by the Renografin flotation method (14, 17).

\section{Isolation of RAA and DEA}

RNA was isolated from the purified kinetoplast-mitochondrion fraction as described (14), using phenol/chloroform ( $1: 1, \nabla / v)$ for deproteinization. The RNA species were separated in $1.5 \%$ agarose-formaldehyde gels, which were blotted onto schleicher and Schuel1 BA 83 nitrocellulose filters for hybridization with ${ }^{32}$ Plabeled nick-translated DNA or oligonucleotide probes 5' endlabeled with 32 P by T4 DNA polynucleotide kinase (16). The hybridizations were performed in probe excess and the amount of kRNA per lane was kept constant. Hybridization conditions were: 0.9 MaC1, 0.09 M Na citrate ( $P$ H 7.0), $0.02 \%$ bovine serum a lbumin, 0.02\% polyvinylpyrol1idine, 0.02\% Fico $11,0.5 \mathrm{mg} / \mathrm{m} 1$ sonicated denatured Salmon sperm DNA, 20 ug/ml E. coli tRNA, $0.05 \%$ Na pyrophosphate, $37 \mathrm{C}, 24 \mathrm{hr}$. The filters were washed in 0.9 MaCl, 0.09 M Na citrate, $0.05 \%$ Na pyrophosphate at $37 \mathrm{C}$ and 45 C. After exposure, the filters were boiled briefly and rehybridized with 32 p-labeled plt120 insert DNA, which contains the $9 S$ and $12 S$ RNA genes in addition to the CYb and COIII genes and several ORFs, to provide internal size standards and to act as a control for extent of degradation (6, 16). For calculation of 
transcript lengths (16), the values of 1200 nt, 1000 nt (12S RNA) and 500 nt ( $9 S$ RNA) were used for the three major RNAs labeled by the 120 probe. The true sizes of the $12 \mathrm{~S}$ and $9 \mathrm{~S}$ ribosomal RNAs are $1160 \mathrm{nt}$ and $610 \mathrm{nt}$, respectively $(7,8)$. However, we have shown previously that the calculated sizes of the $9 \mathrm{~S}$ and $12 \mathrm{~S}$ RNAs from their relative electrophoretic mobility in denaturing methylmercury agarose as compared to several reference RNAs are close to 500 and 1000 nt (14), implying that perhaps denaturation is not complete. The use of these RNAs as internal size markers should therefore be valid. The extent of contamination of the kinetoplast RNA preparations with cytoplasmic ribosomal RNAs was measured by electrophoresis of the RNA in a non-denaturing $1.5 \%$ agarose gel in $90 \mathrm{mM} \mathrm{Tris-HC1,} 90 \mathrm{mM}$ boric acid, $1.5 \mathrm{mM}$ EDTA ( $\mathrm{mH}$ 7.9), $0.5 \mathrm{ug} / \mathrm{ml}$ ethidium bromide. Contamination was generally minimal, as shown by the ratio of the stained 95 and $12 \mathrm{~S}$ RNA bands to the three higher molecular weight cytoplasmic RNA bands in the representative gel shown in Fig. 8.

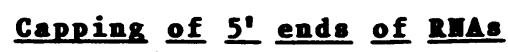

Rinetoplast RNA was 5'-capped with $\alpha-32$-GTP by the method of Levens et a 1 . (18) using guanylytransferase purchased from Bethesda Research Laboratories.

\section{Priner runoffs}

Synthetic oligonucleotides were synthesized using the known DNA sequences (Table I) by the modified triester method (19) and were purifed by gel electrophoresis or HPLC on a Waters c18 column. The primer runoff method of Proudfoot et al (20) was used with the modification that labeling of the runoff products was accomp 1 ished by incorporation of $\alpha-32 p$-dATp during the extension reaction instead of end-labeling the primer. The runoff products were separated on a acrylamide-7M urea $0.2 \mathrm{mmge} 1$ as described previously (6). DNA sequencing ladders of known sequences were co-run as size standards. Reverse transcriptase was purchased from Bethesda Research Laboratories and $\alpha-32$-dATP, $\alpha 32 \mathrm{P}-\mathrm{dGTP}$ and $Y-{ }^{32} \mathrm{P}-\mathrm{ATP}$ from New England Nuclear Company.

\section{RESULTS AID DISCUSSIOI}

\section{Identification of specific transcripts}

The identification of transcripts of specific genes was made 


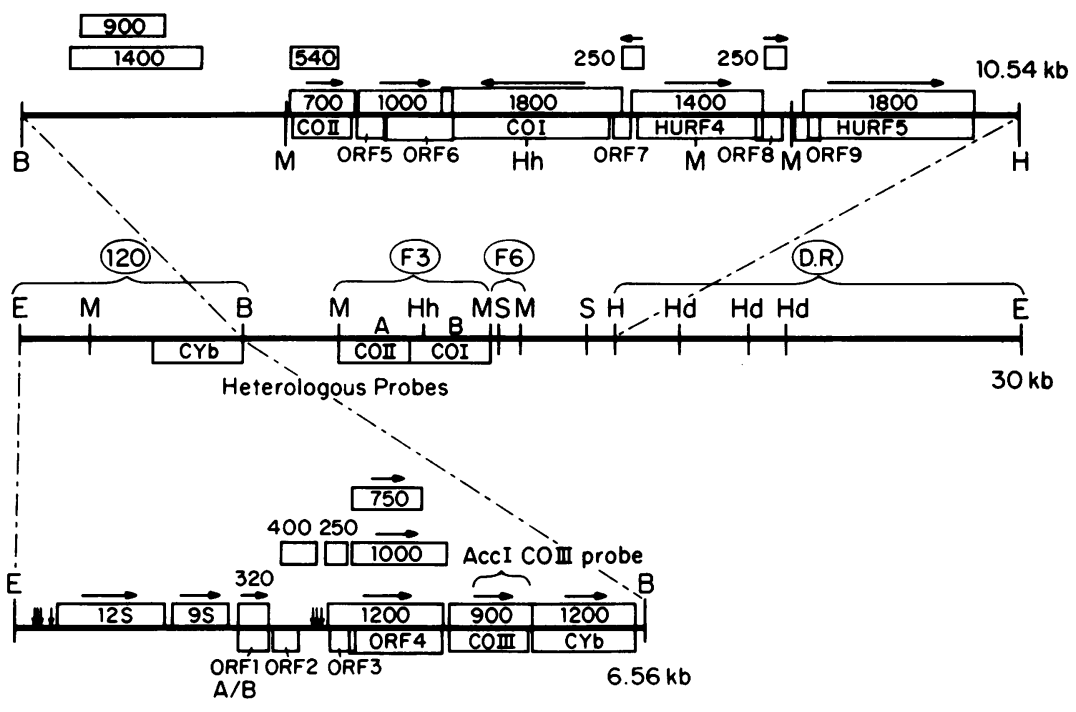

Figure $\underline{1}$

Partial transcription map of L. tarentolae maxicircle. The restriction map of the entire EcoRI linearized maxicircle is shown in the center with the relevant cloned and sequenced fragments indicated by brackets. The non-transcribed divergent region (DR) is a 180 indicated (2). Beneath the center map are presented the approximate localizations of the structural genes as determined previous $1 \mathrm{y}$ by heterologous hybridizations (21). The "120 region" is expanded on the bottom and the remaining "F3F6F1" (refers to the EcoRI/BamHI/MspI maxicircle fragments) transcribed region is expanded on the top, with the identified genes and ORFs indicated beneath the 1 ines and the well localized transcripts on the 1 ine. Poorly localized transcripts are indicated in some cases by boxes above the 1 ine. On 1 y major RNA species are shown on this map since the origins and localizations of the minor species have not yet been determined. The direction of transcription if known is shown by an arrow. The vertical arrows indicate the location of putative tRNA genes. The transcript localizations were derived from the previously published Northern blot analysis using restriction fragment probes (16) and from the present blot analysis and primer runoff analysis.

possible by the previous sequence analysis that led to the identification of maxicircle genes by homology at the amino acid level with mitochondrial genes from other organisms (6). A genomic and transcriptional map is shown in Fig. 1. The transcripts were localized by hybridization of kinetoplast RNA blots with cloned maxicircle restriction fragments (14, 16) and with labeled oligonucleotide primers synthesized according to the known DNA 
Table 1 .

01 igonucleotide Primers used for Primer Runoffs.

\begin{tabular}{|lrlll|}
\hline Primer & \multicolumn{2}{c}{ Location $^{2}$} & Gene & Sequence $\left(5^{\prime}-3^{\prime}\right)$ \\
\hline S5 & F3 & $3324-3338$ & COI & CCTGAAAGGATGCA \\
S6 & 120 & $3396-3381$ & CYB & TGTACTACGTATCAC \\
S8 & F3 & $237-223$ & COII & AAGCAGTGAAGTCTC \\
S9 & 120 & $2409-2393$ & COIII & ACTACCAAACATAATAC \\
S11 & 120 & $3307-3287$ & CYB & ATGCTAAGCAAACACCACATA \\
S12 & F3 & $242-222$ & COII & CTTAGAAGCAGTGAAGTCTCA \\
S15 & F3 & $3823-3804$ & HURF4 & CTGCAAGGCTAAACAAAAAC \\
S16 & F3 & $1156-1136$ & ORF6 & GAACAAAATATAACATTAAAC \\
S17 & F3 & $5604-5585$ & HURF5 & ACATAAAACCACTGACAACC \\
S19 & 120 & $1375-1357$ & ORF4 & CGTATTGCTAGACAGCATC \\
\hline
\end{tabular}

a Location of primer is indicated in th in the respective database ("120" or "F 3 ") as presented in reference 6.

sequences. The oligonucleotides used and the locations in the maxicircle sequence are shown in Table I. The Northern blots shown in Fig. 2 demonstrate that several genes (HURF4, HURF5, ORF4 and ORF5-6) are associated with single RNA species of 1400 , 1800,1200 and 1000 nt, respectively. The probes for coII, coIII, and $C Y b$, however, each hybridize with several RNA species. The COII probe hybridizes with a major 700 nt RNA and a minor 540 nt RNA, the COIII probe with a major 900 nt RNA and minor 2000, 1000,780 , and 530 nt RNAs, and the CYb probe with a major 1200 nt RNA and a minor 500 nt RNA.

The relevance of the minor species is not known; they may represent non-specific hybridization to RNAs from other maxicircle regions or may in some cases represent processing intermediates or degradation. The coll transcript was investigated further by hybridization of the Northern blot with a labeled 644 bp AccI subclone of the 120/Sau3A fragment 1 , which covers most of the 855 bp COIII gene (nt 2493-3136 in the 120 sequence from ref. 6). As shown in Fig. 3, this probe hybridizes with the 900 and 780 nt RNA species only, indicating that the 2000 nt, 1000 nt 


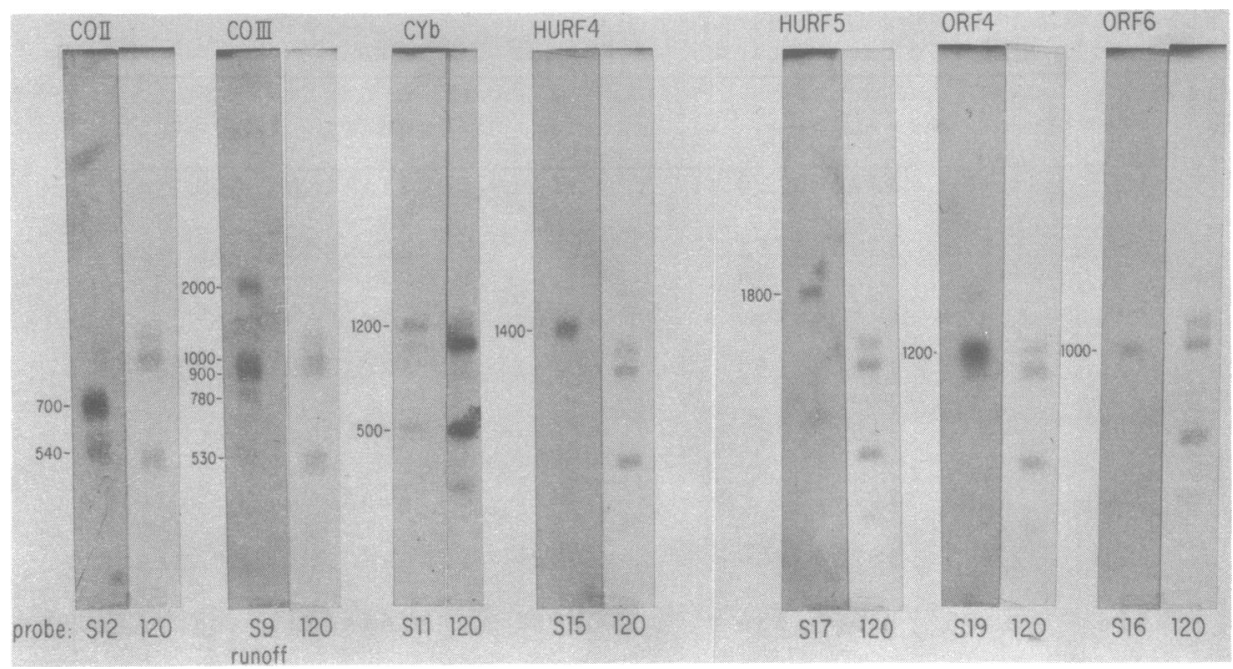

\section{Figure 2}

Northern blot analysis of maxicircle gene transcripts using oligonucleotide probes. Rinetoplast RNA was electrophoresed through formaldehyde agarose, blotted onto nitrocellulose filters and probed with $32_{\mathrm{P}-5}$-end-labeled oligonucleotides specific for individual maxicircle genes and ORFs (Table 1). After exposure, the filters were boiled briefly and rehybridized with 32 p-labeled 120 insert as an internal control for transcript sizes and lack of degradation (16). The probe used for the COIII filter was the 32 p-labeled runoff product from a reaction using the s9 primer with kinetoplast RNA and not the end-labeled primer itself.

and 530 nt RNAs are not transcripts of the COIII gene, but are probably due to nonspecific hybridization of the oligonucleotide primer and the primer runoff, possibly due to the lower stringency of hybridization and wash. This is in agreement with our previously published Northern blot results (Fig. 6b in ref. 16) using $120 /$ Sau $3 A$ restriction fragment $1 \mathrm{~A}$, which covers a lmost the complete corII gene and the 5' portion of the Crb gene. Use of this probe showed no 2000 nt RNA species hybridizing but did show the 1200 nt CYb RNA and the 900 nt COIII RNA in addition to a minor 750 nt RNA species (16).

Evidence for the localization of the 1800 nt col transcript and the specificity of the COI oligonucleotide primer is presented below.

The presence of a major single 1800 nt HURF transcript is 


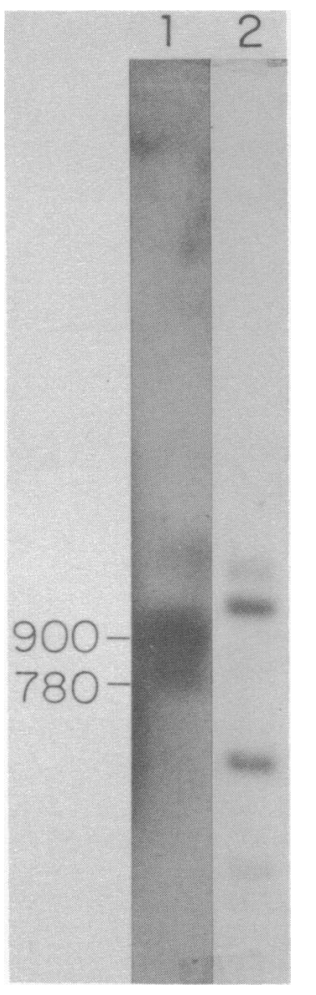

\begin{abstract}
Figure $\underline{3}$
Northern blot analysis of colli gene transcripts using cloned DNA probes. Rinetoplast RNA was electrophoresed through formaldehyde agarose and blotted onto a nitrocellulose filter. In lane 1 the filter was probed with a nicktranslated gel-purified insert from a plasmid which contained a 644 bp AccI fragment specific for the coIII gene (Fig. 1). In lane ${ }^{2}$, the filter. was boiled and reprobed with $3^{2}$ p-labeled 120 insert.
\end{abstract}

consistant with our previously published Northern blot analysis (Fig. 7b in ref. 16) using the maxicircle EcoRI/Mspl restriction fragment 1, which covers the entire HURF5 gene and ORF9. This ana 1 ysis showed a major 1800 nt RNA and in additiona variety of smaller minor RNA species $(1400,1200,1100,850,450,350,250$ nt). In addition, Muhich et al (2) showed that the transcription of the Fl region is 1 imited to the $2.4 \mathrm{~kb}$ HaelI subfragment (See Fig. 1). The nature and role of these minor RNA species is unknown. They may represent nonspecific hybridization of the ge 1isolated probes to RNAs from other regions or may represent processing intermediates or specific breakdown products.

\title{
Strandedness of transcripts
}

We have shown previously that all transcripts from the 120 region are encoded by the same strand as the $9 S$ and $12 S$ RNAs, and this was confirmed for the ORF4, corII and Crb transcripts by the single stranded oligonucleotide hybridizations in Fig. 2. The stranded- 


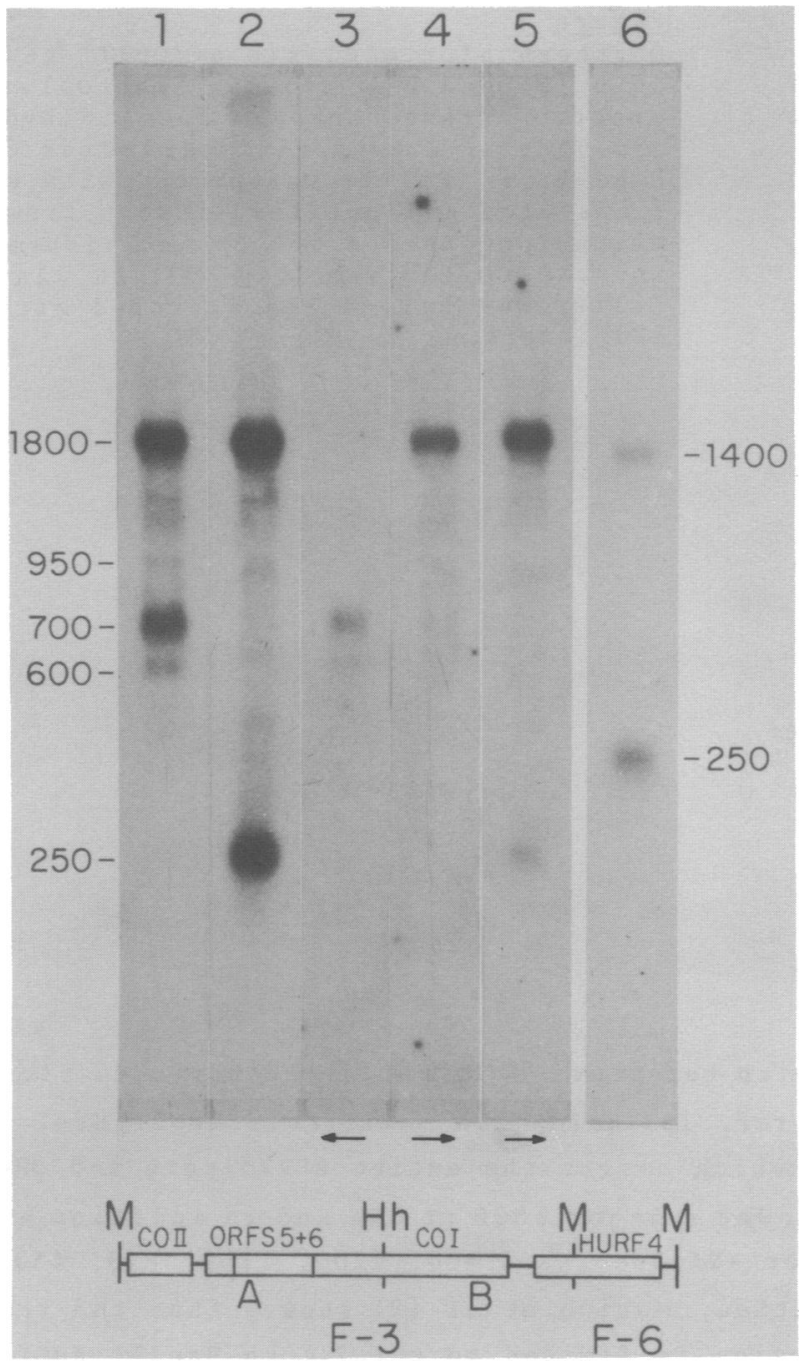

Figure $\underline{4}$

Northerń blot analysis of the F3-F6 region using double stranded (ds) and single stranded (s8) DRA probes. The relevant regions of the maxicircle are shown in the map beneath the autoradiographs ( $M=$ MspI, Hh= HhaI). Orientations of the ss probes are indicated by arrows beneath the lanes. Lane 1, F 34 ds nick-translated probe (2.53 kb insert). Lane 2, F3B ds nick-translated probe (1.78 kb insert). Lanes 3 and 4 , F $3 A 88$ M13 probes of different orientations. Lane 5, F3B 88 M 13 probe. Lane 6, F6 ds nicktranslated probe (0.99 kb insert). The single stranded probes were generated by second strand synthesis of the relevant M13 templates using an M13 universal sequencing primer (15-mer from New Bngland Biolabs). 
\begin{tabular}{l|l|l|l|l|l|l|l|}
\hline A COII & 5 & 6 & COI & & HURF 4 & $\square$ \\
\hline
\end{tabular}

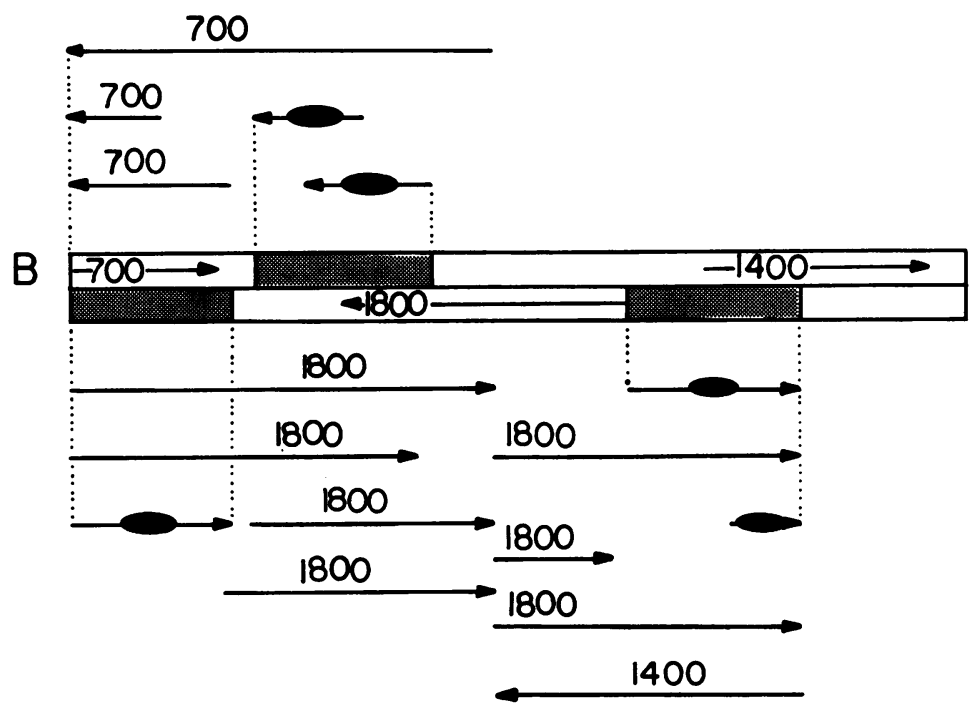

Figure $\underline{5}$

Diagram of results of additional Northern blot analysis of the F3 region. A variety of 88 DNA probes were tested to verify the orientation and to more precisely localize the Cor, corI and HURF 4 gene transcripts. The locations of the genes, as determined by sequence analysis (6), are shown for reference in the map in $A$. The results are summarized in $B$, which represents the two strands of DNA coding for COII, COI and HURF4. Single stranded probes of the indicated polarity which hybridized with the 700 nt COII RNA, the 1800 nt COI RNA or the 1400 HORF 4 RNA are represented by arrows, with the length of each arrow indicating the region covered by the probe, and the transcript to which the probe hybridized indicated by a number above the arrow. Arrows with a black spot represent probes which did not hybridize to any transcript. Note that the ORF5-6 transcript is too low in abundance to be visualized in these blots. The approximate start and stop points for the 700 and 1800 nt RNAs and the start point for the 1400 nt RNA could be determined from these hybridization data, as shown by the labeled arrows within the clear boxes in $B$. The shaded areas represent the lack of hybridization with these strand-specific probes.

ness and localizations of the coII and CoI transcripts were determined by Northern blot analysis (Fig. 4) and by the additional hybridization results diagrammed in Fig. 5. The 700 nt COII RNA is transcribed from the same strand as the 120 RNAs, whereas the 1800 nt COI RNA is transcribed from the opposite strand, as expected from the direction of the col reading frame. 
Nucleic Acids Research

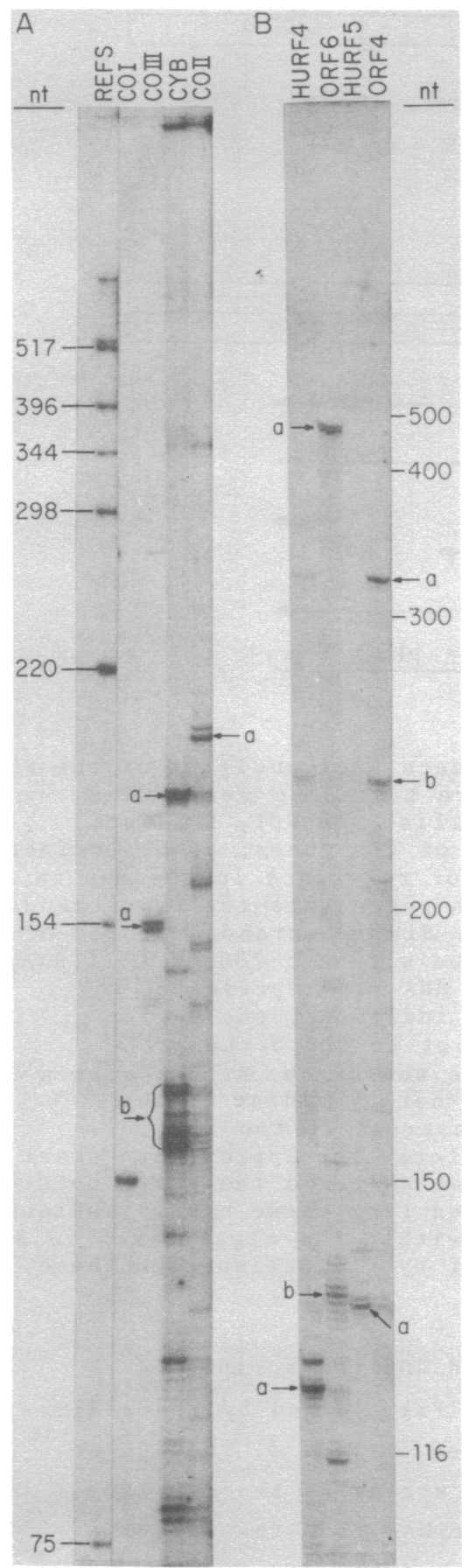

Figure 6

Primer runoffs of maxicircle RNAs for $5^{\prime}$ end localizations. Labeled primer runoffs were obtained as described in Materials and Methods and sized on a $6 \%$ acry 1 amide-7M urea ge 1. In ge $1 \mathrm{~A}$, end-1abeled PBR322/ Hinf 1 fragments were included in lane 1 as size standards. Major (a) and minor (b) runoff products are indicated by arrows. The labeled material at the origin in the CYb and COII lanes probably represent non denatured products. 
The 1400 nt HURF4 RNA and the 1800 nt HURF 5 RNA are transcribed from the same strand as the coll gene in the 33 region.

Localization of $5^{\prime}$ ends of RAs by priner runoff

Synthetic oligonucleotides (Table 1) were used to localize the 5 ' ends of individual transcripts by primer runoff analysis as shown in Fig. 6. The runoff products were co-run next to DNA sequencing ladders which allowed size determinations to be made a lmost to the nucleotide in most cases. The COI runoff product was a single band, whereas the other primers yielded more complex patterns with one or two major and several minor bands. The nature of the minor bands is unknown; they may be due to nonspecific hybridization of the primers to other RNA species or may represent heterogeneous $5^{\prime}$ ends of intermediates in processing or degradation of the primary transcripts. They could also be the result of pausing of reverse transcriptase on the RNA template. The localizations of the major runoff products in the DNA sequence are presented in Fig. 7. Assuming that the major runoff product in each case represents the stable mRNa and insome cases possibly the unprocessed primary transcript, the distance of the 5 ' ends of the RNAs to the putative translation initiation codons (where this can be determined) varies from 20 to 64 nt for the different genes. This differs from the situation in the human mitochondrion where the $5^{\prime}$ ends of the stable mRNAs coincide with the translation initiation codons of the genes, and is more similar to the situation in the yeast mitochondrion where there are multiple promotors and substantial $5^{\prime}$ untranslated sequences. However, the L, tarentolae maxicircle genome is more compact than the yeast mitochondrial genome and the genomic organization of known structural genes differs substantially.

The ORF4 major runoff product localizes the 5' end of this transcript 20-24 nt upstream of the initial amino acid codon in the ORF 3 reading frame. This localization, combined with the 1200 nt size of the transcript as determined by the Northern blot in Fig. 2, suggests that there is a single transcript for ORF3 and ORF4. There is a secondary runoff product (band b, Fig. 6B) of the ORF4 primer that could represent the 5 ' end of a processed ORF3-4 RNA or a reverse transcriptase pause point. Our previousiy published Northern blot results (Fig. 6a in ref. 16), using 120/Sau3A TaqI 
1. ORF3/ORF4 5' END:

LtORF3

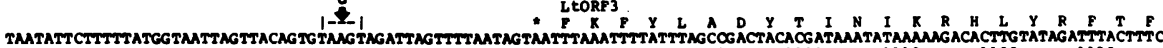

1020

1-c-1

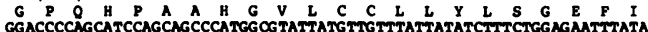

$1140 \quad 1150 \quad 1160 \quad 1170 \quad 1180 \quad 1190 \quad 1200$

2. COIII 5' END:

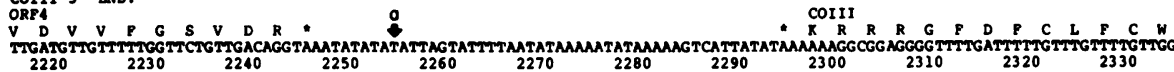

3. CYB 5' END:

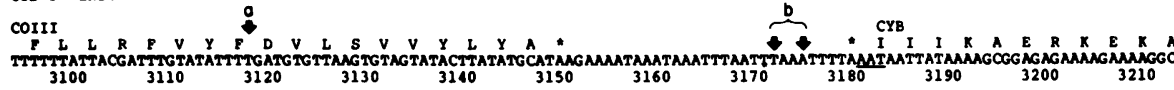

4. CoII 5. END: $\quad$ LtcoII

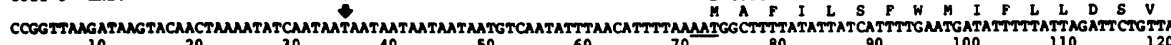

5. ORF5-6 5' END:

COII C G V L H G P A P I V I I

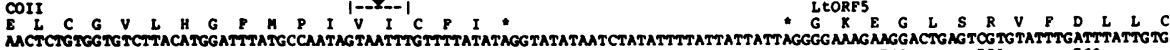

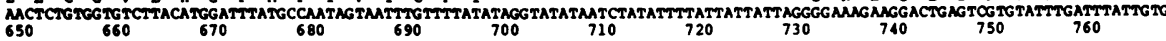
Y I G Y F I Y Y I Y I L Y N F I C Y F F C F S Y C L L Y Y I E F F T Y L L C F I F

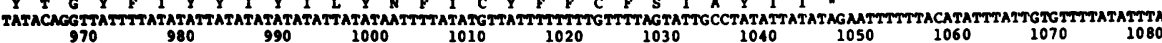

6. $\cos 5^{\prime} \mathrm{EDt}$

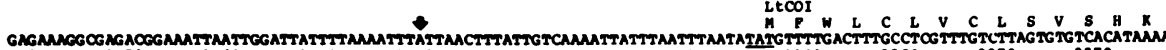

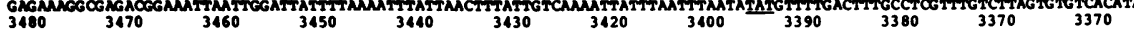

7. HURP4 5' EAD:

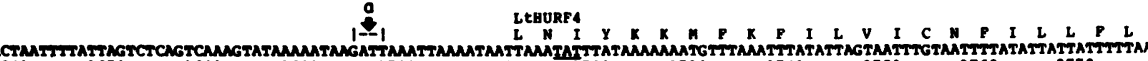

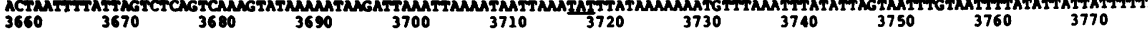

8. MORFS 5 ' EID:

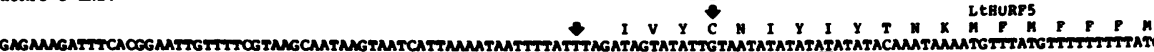
50 (5)

Figure 7

Localization of 5 ' ends of primer runoff products on DNA sequence. The sequences are taken from ref. 6 and the numbering refers to the published 120 region or F 3 region. Open reading frames are indicated by single letter amino acid codes and termination codons by stars. The particular gene is indicated above each ORF and the putative translation initiation codon is indicated by underlining in each case where it can be determined (6). The $5^{\prime}$ ends of the runoff products from the experiment in Fig. 6 are shown by vertical arrows.

restriction subfragments B and $A$ as probes, agree well with the localizations of the 320 nt ORF1 RNA and the 1200 nt ORF3-4 RNA in Fig. 1, provided the corrected TaqI sites are used as determined from the sequence (16).

A similar situation is seen with the ORF6 primer runoff. The size of the major product localizes the $5^{\prime}$ end of the transcript approximately 41-47 nt upstream of the initial amino acid codon in the ORF5 open reading frame. The 1000 nt size of the transcript 
identified by the Northern blot is consistent with a single RNA covering both ORF5 and ORF6. This RNA is a low abundance species compared to the COII and COI transcripts, as shown by the Northern blot in Fig. 4 ( 1 ane 1) and also by the previously published Northern blot in Fig. 7, 1 ane $3 B$ of ref. 16. In the T. brucei maxicircle there is a single continuous ORF homologous to the L. tarentolae ORF5 and ORF6 (10). This implies that the L. tarentolae ORF5 and ORF6 may represent a single gene which has a frameshift anomaly as found previously for corl in both $\underline{\text { s. }}$ tarentolise (6) and T. brucei (10).

The multiple minor small runoff products seen with the sll crb primer (labeled "b" in Fig. 6A) are not due to nonspecific hybridization, since the same bands were seen using another primer (s6 in Table 1) which hybridized to a different site in the Crb gene. However, some non-specific hybridization is indicated by the Northern blot in Fig. 2, in which the CYb primer hybridizes with a 500 nt RNA in addition to the predominant $1200 \mathrm{nt}$ CYb transcript. Our previous ly published (Fig. 6B of ref. 16) blot hybridization using the $120 /$ saus restriction fragment under stringent conditions clearly indicated on $1 y$ a single 1200 nt cybspecific RNA species. The multiple minor bands in the runoff experiment coincide with a 30 nt stretch of pure A+T DNA immediately upstream of the initial amino acid codon of the Crb open reading frame (nt 3160-3180 in the 120 sequence from reference 6) which may be acting as reverse transcriptase pause points.

The HURF5 primer yielded a single runoff product which maps, as shown in Fig. 7 , coincident with the AUA (=isoleucine), which we previously assumed (6) to initiate this open reading frame.However, an inspection of the alignments of the L. tarentolae amino acid sequence with the human and $\underline{T}$. brucei mitochondrial sequences of this gene suggests that the initial translated codon might be the AUA at nt 5479 or 5485 , or the AUG at nt 5500 in the F3 sequence (6), thus localizing the $5^{\prime}$ end 19-30 nt from the initiating codon.

labeling of prinary transcripts of eaxicircle DA

A purifed kinetoplast RNA preparation, which was shown to contain relatively little cytoplasmic ribosomal RHA (Fig. 8) and there- 


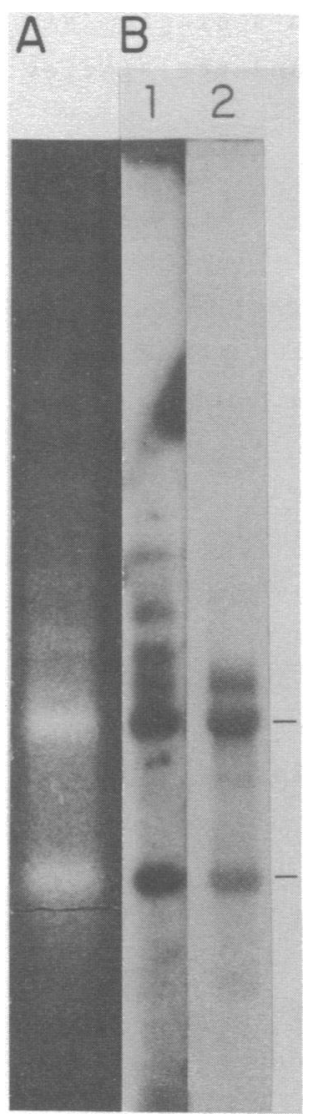

Figure 8

Capping of kinetoplast RNAs by guanylyltransferase and $\alpha-32$-GTP. The ethidium bromide stained agarose gel in $A$ shows the low level of contamination of the kinetoplast RNA preparation with cytoplasmic ribosomal RNAs. The two major species represent the $9 \mathrm{~S}$ and $12 \mathrm{~S}$ kinetoplast RNAB. The autoradiograph in $B$, lane 1 , represents $5^{\prime}$ capped kinetoplast RHA run in a formaldehyde agarose gel. Lane 2 shows the same filter after rehybridization with labeled 120 insert probe and brief exposure to provide an internal size control.The locations of the $9 \mathrm{~S}$ and $12 \mathrm{~S}$ RMAs are indicated by lines.

fore even less cytoplasmic mRHA, was labeled with $\alpha-32$ p-GTP by the enzyme, guanylyltransferase. This enzyme specificaliy recognizes RNA with 5' di- or triphosphates, which are characteristic of unprocessed primary transcripts. The labeled RNAs were separated in a denaturing gel and atoradiographed. As shown in Fig. 8, the major labeled species comigrated with the $9 \mathrm{~s}$ and $12 \mathrm{~S}$ maxicircle RNAs. In addition there were at least four higher molecular weight species of $4000,1900,1480$ and 1200 nt. Although the labeled RHAs were not characterized further, it is probable that the two major speciesmigrating at 500 and 1000 nt represent $5^{\prime}$ end 1 abeledmaxicircle 95 and $12 S$ RHAs and the minor species represent maxicircle mRNA primary transcripts. This conclusion must be verified by direct sequence analysis of the 
labeled RNAs. These preliminary results, however, suggest that there are separate promotors for the transcription of the 95 and $12 S$ RNAs and possibly several additional promotors for maxicircle mRNA 8 .

A computer search for known prokaryotic and eukaryotic promotor consensus sequences showed that these elements are not present at the standard distances of -10 and -35 nt 5 ' of the presumed initiation sites of mRNA synthesis. The only consistent promotor-like consensus sequence noted was a short AT-rich region immediately adjacent to the presumed mRNA initiation sites as shown in Fig. 7. However, due to the overall high AT composition of the maxicircle sequence, the significance of such TATA-like subsequences is difficult to ascertain by inspection alone.

\section{Iranscription nap}

The current transcription map of the sequenced regions of the $\underline{L}$. tarentolae maxicircle is shown in Fig. 1. The localizations were derived both from the previously published (16) Northern blot experiments using labeled maxicircle restriction fragments, and from the above experiments using labeled oligonucleotide probes (Figs. 2-5) and primer runoff localization of 5 ' ends (Figs. 6,7). Sl nuclease protection analysis was also performed for the transcripts of the 120 region (de la cruz, simpson and simpson, unpublished results) and the results agreed well with the localizations in Fig. 1.

In the 120 region, the 320 nt RHA was localized previously (16) by hybridization using the $120 /$ sau3A fragment 7 probe. This RAA corresponds in location and polarity to ORF1A/B. This localization is of some interest in view of the fact that the ORF1A/B sequence does not show the T/A strand bias characteristic of all the other identified maxicircle genes (6). The ORF 2 region is not well analyzed, and the exact 10 cation of the 400 nt and 250 nt RNAs is not known. The vertical arrows downstream of ORF 2 indicate sites where putative tRNA cloverleaf structures can be constructed from the DNA sequence (data not shown). Additional sites are indicated by vertical arrows upstream of the 125 RNA gene. A 1200 nt RNA apparent $1 y$ is the transcript for both ORF 3 and ORF4, a 900 nt RHA is the transcript for coIII, and another 
1200 nt RNA is the transcript for CYb.

In the F3-F6-F1 region, the 700 nt COII RNA, the 1000 nt low abundance ORF5-6 RNA, the 1800 nt COI RNA, the 1400 nt HURF4 RNA, and the 1800 nt HURF 5 RNA are the apparent stable gene transcripts. However, there are several additional RNAs present which have not been well localized. These may represent processing intermediates or may eventually be assigned to other maxicircle transcriptional units.

\section{ACK OWLEDGBHEETS}

This work was supported in part by NIH Grant AI 09102 to LS. VC and MM were predoctoral trainees on NIH Grant GM-7104.

*Present address: Emory University School of Medicine,Department of Biochemistry, Atlanta, GA 30322

+ To whom correspondence and reprint requests should be addressed.

\section{REPERETCES}

1. Simpson, L. (1985) Intern. Rev. Cyto1. In press.

2. Muhich, M., S impson, L. and Simpson, A.M. (1983) Proc. Nat 1. Acad. Sci. USA 80,4060-4064.

3. Borst, P., Fase-Fowler, F., Hoeijmakers, J. and Frasch, A. (1980) Biochim. Biophys. Acta. 610, 197-210.

4. Borst, P., Weijers, P. and Brakenhoff, B. (1982) Biochim. Borst, P., Weijers, P. and Br.
Biophys. Acta $699,272-280$.

5. Muhich, M., Neckelmann, N. and Simpson, L. (1985) Nucl. Acids Res. in press.

6. de la Cruz, V.F., Neckelmann, N. and Simpson, L. (1984) J. Bio 1. Chem. 259, 15136-15147.

7. de 1 a Cruz, V.F., Lake, J.A., Simpson, A.M. and Simpson, L. (1985) Proc. Nat. Acad.Sci. OSA 82, 1401-1405.

8. de la Cruz, V.F., Simpson, A., Lake, J. and Simpson, L. (1985) Nuc1. Acids Res. 13, 2337-2356.

9. Benne, R., DeVries, B., Van den Burg, J. and Klaver, B. (1983) Nuc1. Acids Res. 11, 6925-694i.

10. Hensgens, A., Brakenhoff, J., De Vries, B., sloof, P., Tromp, M., Van Boom, J. and Benne, R. (1984) Nucl. Acids Res. 12, 7327-7344.

11. Johnson, B., Hil1, G. and Donelson, J. (1984) Mol. Biochem. Parasito1. $13,135-146$.

12. Hoeijmakers, J., Snijders, A., Janssen, J. and Borst, P. (1981) Plasmid $5,329-350$.

13. Hoeijmakers, J. and Borst, P. (1982) Biochim. Biophys. Acta. 521, 407-411.

14. Simpson, L. and Simpson, A. (1978) Cel1 14, 169-168.

15. Stuart, R. and Gelvin, S. (1982) Mol. Cel1. Biol. 845-852. 
16. Simpson, L., Simpson, A. and Livingston, L. (1982) Mol. Biochem. Parasito1. 6 , 237-257.

17. Simpson, L. and Braly, P. (1970) J. Protozoo1. 16, 511516 .

18. Levens, D., Ticho, B., Ackerman, E. and Rabinowitz, M. (1981) J. Bio1. Chem. 256, 5226-5232.

19. Ita, H., Ike, Y., Ikuta, S. and Itakura, R. (1982) Nuc1. Acids Res. 9, 2989-2998.

20. Proudfoot, N., Shander, M., Manley, J., Gefter, M. and Maniatis, T. (1980) Science 199, 1329-1336.

21. Simpson, L., Spithill,T. and Simpson, A.M. (1982) Mol. Biochem. Parasitol. $6,253-263$.

22. Hughes, D., Simpson, L., Kayne, P. and Necke1mann, N. (1984) Mo1. Biochem. Parasito1. 13, 263-275. 